\title{
Metrics of the normal anterior sclera: imaging with optical coherence tomography
}

\author{
Andreas Ebneter $^{1}$ - Nathanael U. Häner ${ }^{1}$ Martin S. Zinkernagel ${ }^{1}$
}

Received: 13 October 2014 / Revised: 17 May 2015 / Accepted: 25 May 2015 / Published online: 12 June 2015

(C) The Author(s) 2015. This article is published with open access at Springerlink.com

\begin{abstract}
Background To investigate anterior scleral thickness in a cohort of healthy subjects using enhanced depth imaging anterior segment optical coherence tomography.

Methods Observational case series. The mean scleral thickness in the inferonasal, inferotemporal, superotemporal, and superonasal quadrant was measured $2 \mathrm{~mm}$ from the scleral spur on optical coherence tomography in healthy volunteers. Results Fifty-three eyes of 53 Caucasian patients (25 male and 28 female) with an average age of 48.6 years (range: 18 to 92 years) were analysed. The mean scleral thickness was $571 \mu \mathrm{m}$ (SD $84 \mu \mathrm{m})$ in the inferonasal quadrant, $511 \mu \mathrm{m}$ (SD $80 \mu \mathrm{m})$ in the inferotemporal quadrant, 475 (SD $81 \mu \mathrm{m})$ in the superotemporal, and 463 (SD $64 \mu \mathrm{m})$ in the superonasal quadrant. The mean scleral thickness was significantly different between quadrants $(p<0.0001$, repeated measures one-way ANOVA). The association between average scleral thickness and age was statistically significant $(p<0.0001$, Pearson $r=$ $0.704)$.

Conclusions Enhanced depth imaging optical coherence tomography revealed the detailed anatomy of the anterior sclera and enabled non-invasive measurements of scleral thickness in a non-contact approach. The anterior scleral thickness varies significantly between quadrants, resembling the spiral
\end{abstract}

Electronic supplementary material The online version of this article (doi:10.1007/s00417-015-3072-5) contains supplementary material, which is available to authorized users.

Andreas Ebneter

ebneter.andreas@gmail.com

1 Department of Ophthalmology, Inselspital, Bern University Hospital, and University of Bern, Bern, Switzerland of Tillaux. An association of increasing scleral thickness with age was found.

Keywords Anterior segment OCT · Imaging · Sclera · Age · Intravitreal injection · Glaucoma

\section{Introduction}

The sclera is the tension-bearing component of the eye, representing the cornerstone and prerequisite for permanent and stable shape. Despite its bradytrophic nature and low cellular content [1], evidence has emerged in recent years that the sclera may play a major role in ocular disease and pathology [2]. While the focus of investigations has mainly been its posterior part to this point [3-5], the scientific community becomes more attracted to the anterior portion because of the popularity of intravitreal injections, the potential role in alternative transscleral drug delivery [6], new anterior segment operating techniques [7], and the role of the trabecular meshwork in glaucoma [8].

Scleral properties are central in various aspects of glaucoma development and treatment. Biomechanics of the sclera are considered paramount in the pathogenesis of at least some types of glaucomas [9]. Both material properties, such as stiffness, and anatomical features determine the distribution of mechanical stress at the optic nerve head and the lamina cribrosa [10], the putative site of the initial glaucomatous insult $[11,12]$. Although not the rate-limiting step, scleral conductivity is a determinant of uveoscleral outflow that is modified by prostaglandin analogues [13]. However, the exact role of the anterior sclera in the pathogenesis of glaucoma is unknown.

Optical coherence tomography (OCT) has become very popular, and has evolved quickly for imaging of the posterior 
segment. This non-contact method provides high-resolution images of the retina and adjacent structures. The newly available enhanced depth imaging (EDI) modules allow even more in-depth analysis of the choroid and sclera [14]. More recently, OCT has become available for non-invasive assessment of the anterior segment [15]. To date, the focus has primarily been the cornea and anterior chamber structures and dimensions [16]. The aim of the current work was to characterize the sclera adjacent to the limbus using EDI-OCT in healthy eyes.

\section{Materials and methods}

\section{Subjects}

This study was designed as a prospective observational case series. Healthy Caucasian volunteers over the age of 18 years attending the outpatients clinic at a tertiary hospital (Inselspital Bern, Switzerland) were recruited from December 2012 to December 2013. A detailed ocular and general medical history was obtained, and participants underwent a comprehensive slit-lamp examination including intraocular pressure measurement. Individuals with systemic disease potentially affecting the eye (including diabetes mellitus, thyroid disease, and systemic inflammatory conditions) were not eligible. Eyes with previous surgery or signs of relevant ocular pathology such as glaucoma, keratoconus, or high myopia (axial length> $26.5 \mathrm{~mm}$ ) were excluded.

All procedures performed involving human participants were in accordance with the ethical standards of the institutional research committee and with the 1964 Helsinki Declaration and its later amendments or comparable ethical standards. The Bern University Institutional Review Board and the ethics committee had granted approval (KEK 178/ 12) for the project. Written informed consent was obtained from all individual participants included in the study.

\section{Imaging}

The axial length was measured using the IOL-Master 500 (Carl Zeiss Meditec, Oberkochen, Germany). Spectraldomain optical coherence tomography (SD-OCT) of the perilimbal sclera was obtained with the anterior segment module (Heidelberg Engineering, Dossenheim, Germany) in sclera mode with EDI. In each quadrant, a volume scan consisting of $21 \mathrm{~B}$-scans was recorded comprising the limbus. The orientation of the individual B-scans was approximately perpendicular to the tangent to the limbus (Fig. 1a). The resolution was $768 \times 496$ pixels $(381 \mathrm{~KB})$ with a scan angle of $15^{\circ}$. For image acquisition in each of the four quadrants (inferotemporal, inferonasal, superonasal, superotemporal) patients were asked to maintain gaze position in the opposite direction to the quadrant examined, and care was taken to include the limbus on all scans. The scleral thickness was independently assessed by two masked graders using the Heidelberg eye explorer software (version 1.7.1.0). For each scan area, the scleral thickness was manually measured $2 \mathrm{~mm}$ from the scleral spur on the three sections that were of best quality using the caliper tool, and the mean was recorded. The $2-\mathrm{mm}$ distance was chosen in order to compare results to previous work by others [17-19]. The episclera and the conjunctiva were not included in the measurement. The external limit of the sclera can be identified by the deep espiscleral vascular plexus, which manifests as a thin hyporeflective space above the solid scleral tissue (Fig. 1b).

\section{Statistical analysis}

All measurements of continuous variables are reported as means (standard deviation). To ascertain independence of data points, only one eye per patient was included if data from both eyes was available. The eye was randomly selected at the time of statistical data analysis based on a list of integer numbers generated using Microsoft Excel for Mac (Version 14.4.2). Repeated measures one-way ANOVA with Tukey's correction for multiple comparison post-hoc tests was used to analyze quadrant scleral thickness data. The correlation between scleral thickness and age was analyzed calculating the Pearson $r$. The best-fit regression line was determined using the leastsquares method for display in scatter plots. The level of significance was 0.05 (two-sided) for all statistical tests. All analyses were performed using commercial software (Prism 6 for Mac OS X, version 6.0d; GraphPad Software, Inc., San Diego, CA, USA).

\section{Results}

OCTs from 53 eyes of 53 healthy volunteers were included in the analysis. The study population consisted of 25 male and 28 female Caucasian individuals. Participants were on average 48.6 years old (median: 47 years, range: 18 to 92 years). The mean age was 49.4 years (median: 47 years, range: 18 to 90 years) for males, and 47.9 years (median: 47 years, range: 18 to 92 years) for females. The demographic information is shown in Table 1. Axial lengths were evenly distributed with age, and no correlation $(p=0.429)$ was found between these two variables (Supplementary Fig 1).

First, we analyzed scleral thickness and its relationship with individual quadrants. We found that scleral thickness in consecutive quadrants decreased steadily $(p<0.0001$, repeated measures one-way ANOVA) from the inferonasal to the superonasal quadrant. Post-hoc multiple comparison confirmed that the scleral thickness varied significantly between all but the superotemporal and superonasal quadrants (Fig. 2a). The mean scleral thickness was 571 (84) $\mu \mathrm{m}$ in 


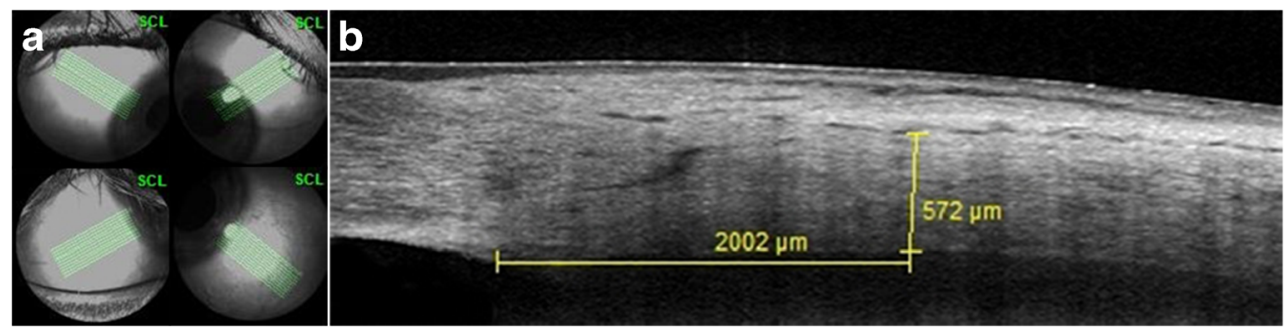

Fig. 1 Anterior segment spectral domain optical coherence tomography scans. The photographs illustrate the scan acquisition (a) and measurement of the anterior scleral thickness $2 \mathrm{~mm}$ posterior to the scleral spur (b). In each quadrant, 21 high-resolution B-scans taken approximately perpendicular to the tangent to the limbus were recorded,

the inferonasal quadrant, $511(80) \mu \mathrm{m}$ in the inferotemporal quadrant, 475 (81) $\mu \mathrm{m}$ in the superotemporal, and 463 (64) $\mu \mathrm{m}$ in the superonasal quadrant.

Next, we studied the association between age and the average scleral thickness. Interestingly, the correlation was highly significant $(p<0.0001$, Pearson $r=0.704)$, average scleral thickness being the arithmetical mean of the scleral thickness in each of the four quadrants (Fig. 2b). This correlation was confirmed ( $p<0.0001$ in each quadrant) when data were reevaluated for each individual quadrant separately (Fig. 3). Data were also analyzed with respect to possible associations between scleral thickness and gender (data not shown). However, no significant relationship was found.

\section{Discussion}

Various techniques have been used in the past to measure scleral thickness [20-24]. Before in-vivo methods became available, investigators examined fixed human donor globes. A fairly consistent picture emerged, and it was confirmed that the sclera is thickest at the posterior pole and the corneoscleral limbus, with its thinnest zone around the equator [21, 22, 25]. The drawbacks of fixed-tissue analysis are susceptibility to post-mortem changes and fixation artifacts. Measurements from fixed tissue were reported to be about $8 \%$ thicker than ultrasound data [22]. Hence, findings might not accurately

Table 1 Demographic data characterizing patients and eyes included in the study

\begin{tabular}{lll}
\hline & Age (SD) [years] & Axial length (SD) [mm] \\
\hline All $(n=53)$ & $48.6(22.9)$ & $23.97(1.68)$ \\
Male $(n=25)$ & $49.4(22.9)$ & $24.13(1.27)$ \\
Female $(n=28)$ & $47.9(23.3)$ & $23.81(1.99)$ \\
$<30(n=18)$ & $24.8(3.1)$ & $23.52(0.91)$ \\
$30-49(n=11)$ & $39.0(7.4)$ & $25.18(2.16)$ \\
$50-69(n=11)$ & $59.5(6.1)$ & $24.17(2.22)$ \\
$>=70(n=13)$ & $80.2(7.5)$ & $23.48(1.16)$ \\
\hline
\end{tabular}

with the patient holding fixation in the direction opposite to the quadrant recorded (a). Scleral thickness was measured between the lamina fusca and the deep episcleral vascular plexus as illustrated in microphotograph b

represent the in-vivo situation. This uncertainty was eliminated when ultrasound biomicroscopy (UBM) and magnetic resonance imaging (MRI) became available. Since the resolution of the latter method was insufficient, UBM turned out to be the preferred choice [20]. However, image acquisition with the UBM technique requires direct contact, and exposure may be challenging because of bulky transducers. The advent of OCT, a non-contact method, has revolutionized posterior segment imaging, not least because of its high resolution, and modifications for anterior segment use have rapidly become available. A plethora of work has been published on applications for cornea, anterior chamber, and trabecular meshwork imaging. Here, we have shown that the anterior segment EDIOCT also represents a practical and convenient method to assess anterior scleral thickness (AST).

The measurements of the AST obtained in this study corroborate findings from previous work with UBM in vivo and post-mortem studies. It is worthy of note that AST is not uniform around the circumference of the globe but varies between quadrants, being thickest in the inferonasal and thinnest in the superonasal quadrant. This pattern is reminiscent of the spiral of Tillaux, describing the insertion of the rectus muscles with respect to the limbus.

Vurgese et al. [25] conducted a study on human donor eyes fixed in formaldehyde and glutaraldehyde. At the limbus a mean scleral thickness of $500 \mu \mathrm{m}$ was reported, similar to our results. In a study by Norman et al. [21] on seven enucleated formalin-fixed human globes measured with high-field microMRI, scleral thickness at the corneoscleral limbus was $588 \mu \mathrm{m}$. Olsen et al. [22] found a mean scleral thickness at the limbus of $530 \mu \mathrm{m}$ in 55 formalin-fixed eyes. Lam et al. [20] published data on the AST measured in four meridians 2$3 \mathrm{~mm}$ posterior to the scleral spur by UBM in ten healthy eyes from five subjects (mean age 67 years). The mean scleral thickness was $550 \mu \mathrm{m}$.

In one of the first UBM studies, Pavlin et al. reported a scleral thickness at the scleral spur of $940 \mu \mathrm{m}$ in nine normal subjects [23]. In a more recent study, 140 eyes of 140 healthy patients were examined with UBM 2 and $3 \mathrm{~mm}$ posterior to the scleral spur in the temporal scleral triangle. The mean AST 


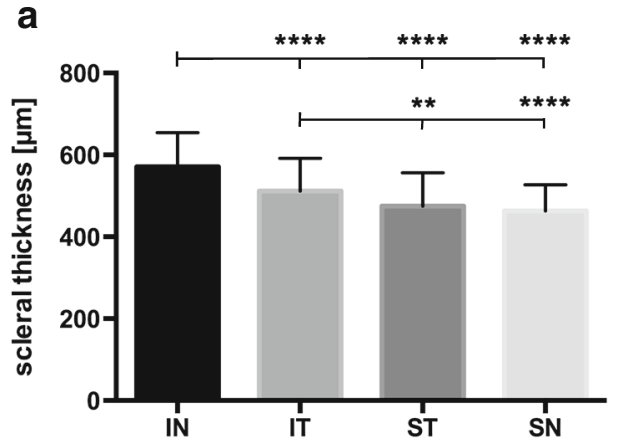

Fig. 2 Influence of quadrant and age on scleral thickness. Graph a shows the mean anterior scleral thickness for each quadrant [inferonasal $(I N)$, inferotemporal $(I T)$, superotemporal $(S T)$, superonasal $(S N)]$. Repeated measures one-way ANOVA was highly statistically significant $(p<0.0001)$. The anterior scleral thickness in the inferonasal quadrant is significantly thicker than in every other quadrant. The anterior scleral thickness in the inferotemporal quadrant too is significantly different from the superonasal and superotemporal quadrant scleral thickness

$2 \mathrm{~mm}$ from the limbus was $511 \mu \mathrm{m}$ [18]. Discrepancies may arise from different definitions of scleral thickness (see below). In a study conducted with the Visante anterior segment OCT, Taban et al. [24] reported a mean scleral thickness of $920 \mu \mathrm{m}$ in normal eyes 3.5 to $4.0 \mathrm{~mm}$ posterior to the scleral spur. They included episclera and conjunctiva in their measurements, which explains some of the difference with this study. Intriguingly, the AST was thickest in the inferonasal quadrant, followed by the inferotemporal, the superotemporal,

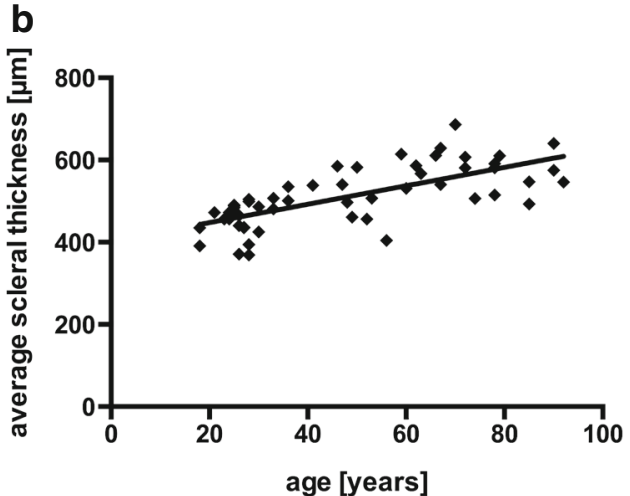

(post-hoc test with Tukey's correction for multiple comparison; ** $p<0.01, * * * * p<0.0001)$. No difference was found between the superonasal and superotemporal quadrants. The scatter plot (b) illustrates the positive correlation between scleral thickness and age $(p<0.0001$, Pearson $r=0.704)$. Average scleral thickness is the mean of all quadrants for each eye. The line represents the best-fit linear regression estimate

and then the superonasal quadrant. We have reproduced this finding.

Interestingly, we also found an association between scleral thickness and age in this cohort of healthy Caucasian volunteers. Age-related changes of human sclera material properties such as loss of compliance have been described by several authors [26, 27], and seem to differ between ethnicities [28, 29]. Yet, in previous reports, an association between AST and age was not evident $[30,25]$. However, all these data were
Fig. 3 Scatter plots for anterior scleral thickness and age in individual quadrants. The correlation between anterior scleral thickness and age was highly statistically significant in each quadrant $[p<0.0001$; inferonasal $(I N)$, inferotemporal (IT), superotemporal (ST), superonasal $(S N)]$. Lines represent the best-fit linear regression estimates [Pearson $r=0.664$ (IN), $r=0.738$ (IT), $r=0.703$ (SN), $r=0.637(\mathrm{ST})]$
IN

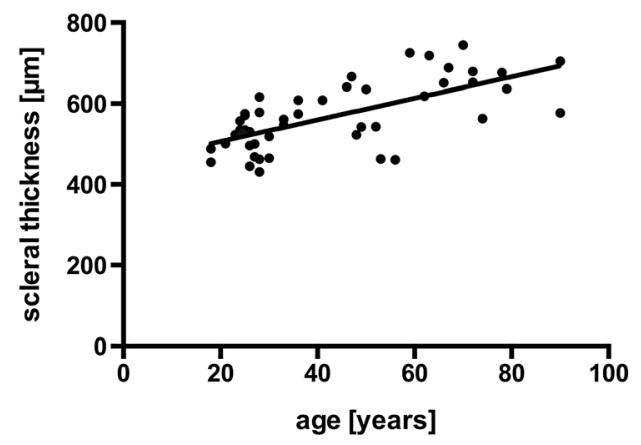

SN

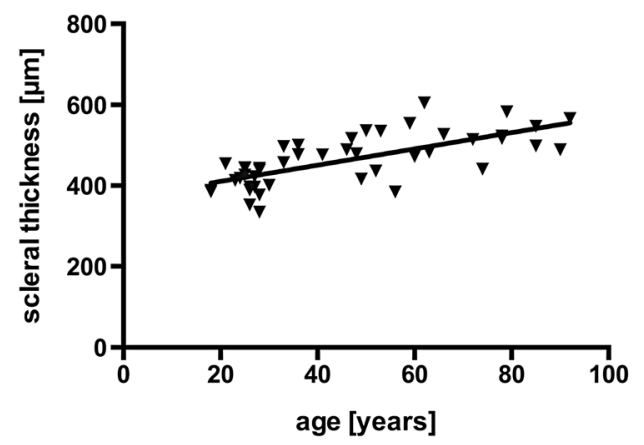

IT

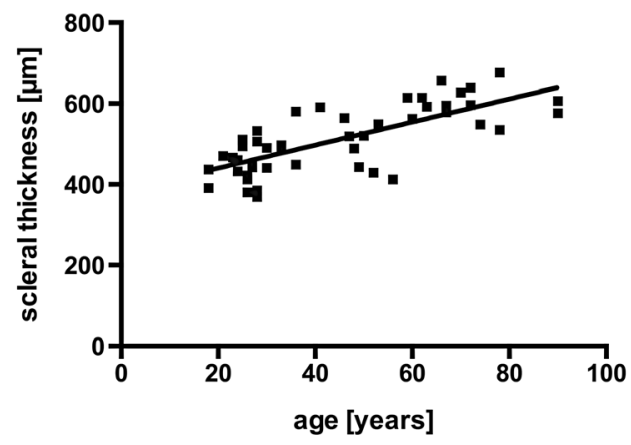

ST

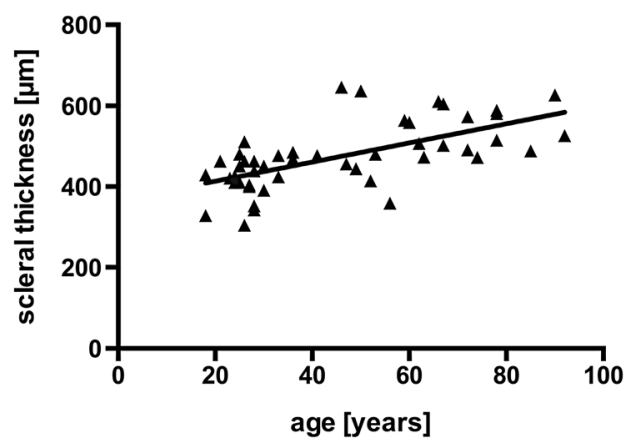


based on post-mortem studies and analysis was not by quadrants. Thickening of the anterior sclera associated with aging found in this report needs to be confirmed in the future, preferably in a population-based longitudinal approach. Participants in this study were recruited in an outpatient eye clinic setting, and selection bias cannot be ruled out.

Knowing scleral thickness of the anterior segment may be of relevance in a number of clinical scenarios. There is some evidence that repeated intravitreal injections in the same quadrant may cause some degree of scleral thinning [31]. Furthermore, scleral thickness is a major determinant of scleral hydraulic conductivity, which determines resistance to transscleral fluid movements, a component of the uveoscleral outflow. Although not the rate-limiting step of uveoscleral outflow under normal circumstances, transscleral resistance does determine outflow in some pathological states [32]. While the posterior sclera seems pivotal in the pathogenesis of glaucoma [33], the significance of the anterior sclera in this disease is not yet clear. Several studies suggest that central corneal thickness, a parameter predicting progression in ocular hypertensive patients [34], is not related to AST [17, 18, 25], and AST may not be as useful in assessing the glaucoma risk. Even so, Yoo et al. found a correlation between central corneal thickness and AST in patients with normal tension glaucoma, but not in primary openangle glaucoma subjects or controls [19]. Mohamed-Noorl et al. reported that temporal AST measured by UBM was thicker in ocular hypertensive patients than in subjects suffering from normal tension glaucoma, but not between other subgroups [17]. In this study, however, we only evaluated healthy eyes, and further research will be needed to elucidate the role of the anterior sclera in the pathogenesis of glaucoma.

There are several advantages of OCT in imaging anterior segment structures. Firstly, due to intrinsic properties of light, the resolution is higher for EDI-OCT compared to soundwave-based methods. Secondly, OCT is a non-contact method and is more practical and convenient than immersion ultrasound. Thirdly, data storage for longitudinal comparison is more straightforward with OCT technology. And lastly, OCT has become more accessible, because it is now routinely used for imaging of the posterior segment. Some devices can be operated in both anterior and posterior segment mode.

The main drawback of EDI-OCT compared to UBM for anterior segment imaging is penetration depth, which precludes examination of the ciliary body. However, some improvement has been achieved with the use of longer wavelengths [35], and most clinically relevant deep anterior segment structures can be visualized on commercial devices [36]. Nevertheless, ultrasound and UBM are still the methods of choice for the assessment of the ciliary body [37]. Tissue differentiation is possible to some degree by standardized echography [38]. The posterior sclera, extraocular muscles, and orbital tissue can be examined with conventional ocular ultrasound. Imaging of the sclera adjacent to the anterior segment with EDI-OCT is feasible to some extent, but can be challenging with the currently marketed OCT devices. Approximately perpendicular exposure to the scan beam is desirable, and the restricted length of the OCT scans may impede simultaneous capture of reference structures, such as the scleral spur. In the current study, specifically, radial scans extending beyond $2 \mathrm{~mm}$ posterior to the limbus with the scleral spur as reference structure could not be obtained consistently. However, these constraints might be obsolete with the advent of hand-held OCT devices that become increasingly accessible [39]. It may be relevant to measure scleral thickness in several locations because of topographic variability, not only antero-posteriorly [40, 21, 25], but also circumferentially, as highlighted in this study.

A difficulty of determining scleral thickness in general is the definition and delineation of the sclera on the images. Some authors measure from the retina to the conjunctival surface, whereas others refer to the anatomical definition and exclude uveal tissue, episclera, and conjunctiva. This is partly because it can be ambiguous to determine the interfaces, particularly when using ultrasound-based systems. A common consensus regarding the definition of these structures for imaging would be useful.

In summary, anterior segment OCT is a convenient and non-invasive method to assess the anterior sclera. Scleral thickness varies between quadrants, and there might be a positive association with age. Further prospective studies are warranted.

Acknowledgments The authors would like to thank Ms Petra Schorno, Ms Bettina Schlatter and Mr Marco Beck for their expert technical assistance in data acquisition and analysis.

Conflict of interest M.S.Z. received financial support from Heidelberg Engineering for posterior segment imaging applications.

All other authors certify that they have no affiliations with or involvement in any organization or entity with any financial interest (such as honoraria; educational grants; participation in speakers' bureaus; membership, employment, consultancies, stock ownership, or other equity interest; and expert testimony or patent-licensing arrangements), or nonfinancial interest (such as personal or professional relationships, affiliations, knowledge or beliefs) in the subject matter or materials discussed in this manuscript.

Open Access This article is distributed under the terms of the Creative Commons Attribution 4.0 International License (http:// creativecommons.org/licenses/by/4.0/), which permits unrestricted use, distribution, and reproduction in any medium, provided you give appropriate credit to the original author(s) and the source, provide a link to the Creative Commons license, and indicate if changes were made.

\section{References}

1. Watson PG, Young RD (2004) Scleral structure, organisation and disease. A review. Exp Eye Res 78(3):609-623 
2. Sainz de la Maza M, Tauber J, Foster CS (2012) The sclera, 2nd edn. Springer, New York

3. Bellezza AJ, Hart RT, Burgoyne CF (2000) The optic nerve head as a biomechanical structure: initial finite element modeling. Invest Ophthalmol Vis Sci 41(10):2991-3000

4. Jonas JB, Holbach L (2005) Central corneal thickness and thickness of the lamina cribrosa in human eyes. Invest Ophthalmol Vis Sci 46(4):1275-1279

5. Quigley HA, Flower RW, Addicks EM, McLeod DS (1980) The mechanism of optic nerve damage in experimental acute intraocular pressure elevation. Invest Ophthalmol Vis Sci 19(5):505-517

6. Lee SB, Geroski DH, Prausnitz MR, Edelhauser HF (2004) Drug delivery through the sclera: effects of thickness, hydration, and sustained release systems. Exp Eye Res 78(3):599-607

7. Doors M, Berendschot TT, de Brabander J, Webers CA, Nuijts RM (2010) Value of optical coherence tomography for anterior segment surgery. J Cataract Refract Surg 36(7):1213-1229

8. Wong HT, Lim MC, Sakata LM, Aung HT, Amerasinghe N, Friedman DS, Aung T (2009) High-definition optical coherence tomography imaging of the iridocorneal angle of the eye. Arch Ophthalmol 127(3):256-260

9. Burgoyne CF, Downs JC, Bellezza AJ, Suh JK, Hart RT (2005) The optic nerve head as a biomechanical structure: a new paradigm for understanding the role of IOP-related stress and strain in the pathophysiology of glaucomatous optic nerve head damage. Prog Retin Eye Res 24(1):39-73

10. Sigal IA, Flanagan JG, Ethier CR (2005) Factors influencing optic nerve head biomechanics. Invest Ophthalmol Vis Sci 46(11):41894199

11. Chidlow G, Ebneter A, Wood JP, Casson RJ (2011) The optic nerve head is the site of axonal transport disruption, axonal cytoskeleton damage and putative axonal regeneration failure in a rat model of glaucoma. Acta Neuropathol 121(6):737-751

12. Quigley HA, Hohman RM, Addicks EM, Massof RW, Green WR (1983) Morphologic changes in the lamina cribrosa correlated with neural loss in open-angle glaucoma. Am J Ophthalmol 95(5):673691

13. Kaufman PL (2008) Enhancing trabecular outflow by disrupting the actin cytoskeleton, increasing uveoscleral outflow with prostaglandins, and understanding the pathophysiology of presbyopia interrogating Mother Nature: asking why, asking how, recognizing the signs, following the trail. Exp Eye Res 86(1):3-17

14. Wong IY, Koizumi H, Lai WW (2011) Enhanced depth imaging optical coherence tomography. Ophthalmic Surg Lasers Imaging 42(Suppl):S75-S84

15. Jancevski M, Foster CS (2010) Anterior segment optical coherence tomography. Semin Ophthalmol 25(5-6):317-323

16. Sharma R, Sharma A, Arora T, Sharma S, Sobti A, Jha B, Chaturvedi N, Dada T (2014) Application of anterior segment optical coherence tomography in glaucoma. Surv Ophthalmol 59(3):311-327

17. Mohamed-Noor J, Bochmann F, Siddiqui MA, Atta HR, Leslie T, Maharajan P, Wong YM, Azuara-Blanco A (2009) Correlation between corneal and scleral thickness in glaucoma. J Glaucoma 18(1): $32-36$

18. Oliveira C, Tello C, Liebmann J, Ritch R (2006) Central corneal thickness is not related to anterior scleral thickness or axial length. $\mathrm{J}$ Glaucoma 15(3):190-194

19. Yoo C, Eom YS, Suh YW, Kim YY (2011) Central corneal thickness and anterior scleral thickness in Korean patients with openangle glaucoma: an anterior segment optical coherence tomography study. J Glaucoma 20(2):95-99

20. Lam A, Sambursky RP, Maguire JI (2005) Measurement of scleral thickness in uveal effusion syndrome. Am J Ophthalmol 140(2): 329-331

21. Norman RE, Flanagan JG, Rausch SM, Sigal IA, Tertinegg I, Eilaghi A, Portnoy S, Sled JG, Ethier CR (2010) Dimensions of the human sclera: thickness measurement and regional changes with axial length. Exp Eye Res 90(2):277-284

22. Olsen TW, Aaberg SY, Geroski DH, Edelhauser HF (1998) Human sclera: thickness and surface area. Am J Ophthalmol 125(2):237-241

23. Pavlin CJ, Harasiewicz K, Foster FS (1992) Ultrasound biomicroscopy of anterior segment structures in normal and glaucomatous eyes. Am J Ophthalmol 113(4):381-389

24. Taban M, Lowder CY, Ventura AA, Sharma S, Nutter B, Hayden BC, Dupps WJ, Kaiser PK (2010) Scleral thickness following fluocinolone acetonide implant (Retisert). Ocul Immunol Inflamm 18(4):305-313

25. Vurgese S, Panda-Jonas S, Jonas JB (2012) Scleral thickness in human eyes. PLoS One 7(1), e29692

26. Coudrillier B, Pijanka J, Jefferys J, Sorensen T, Quigley HA, Boote C, Nguyen TD (2015) Collagen structure and mechanical properties of the human sclera: analysis for the effects of age. J Biomech Eng 137(4):041006

27. Geraghty B, Jones SW, Rama P, Akhtar R, Elsheikh A (2012) Agerelated variations in the biomechanical properties of human sclera. J Mech Behav Biomed Mater 16:181-191

28. Fazio MA, Grytz R, Morris JS, Bruno L, Girkin CA, Downs JC (2014) Human scleral structural stiffness increases more rapidly with age in donors of African descent compared to donors of European descent. Invest Ophthalmol Vis Sci 55(11):7189-7198

29. Grytz R, Fazio MA, Libertiaux V, Bruno L, Gardiner S, Girkin CA, Downs JC (2014) Age- and race-related differences in human scleral material properties. Invest Ophthalmol Vis Sci 55(12):81638172

30. Jackson TL, Hussain A, Hodgetts A, Morley AM, Hillenkamp J, Sullivan PM, Marshall J (2006) Human scleral hydraulic conductivity: age-related changes, topographical variation, and potential scleral outflow facility. Invest Ophthalmol Vis Sci 47(11):4942 4946

31. Zinkernagel MS, Schorno P, Ebneter A, Wolf S (2015) Scleral thinning after repeated intravitreal injections of anti-vascular endothelial growth factor agents in the same quadrant. Invest Ophthalmol Vis Sci 56(3):1894-1900

32. Alm A, Nilsson SF (2009) Uveoscleral outflow-a review. Exp Eye Res 88(4):760-768

33. Campbell IC, Coudrillier B, Ross Ethier C (2014) Biomechanics of the posterior eye: a critical role in health and disease. J Biomech Eng 136(2):021005

34. Gordon MO, Beiser JA, Brandt JD, Heuer DK, Higginbotham EJ, Johnson CA, Keltner JL, Miller JP, Parrish RK 2nd, Wilson MR, Kass MA (2002) The Ocular Hypertension Treatment Study: baseline factors that predict the onset of primary open-angle glaucoma. Arch Ophthalmol 120(6):714-720

35. Li P, An L, Lan G, Johnstone M, Malchow D, Wang RK (2013) Extended imaging depth to $12 \mathrm{~mm}$ for $1050-\mathrm{nm}$ spectral domain optical coherence tomography for imaging the whole anterior segment of the human eye at $120-\mathrm{kHz}$ A-scan rate. J Biomed Opt 18(1):16012

36. Theelen T, Hoyng CB (2013) A prospective, comparative, observational study on optical coherence tomography of the anterior eye segment. Ophthalmologica 230(4):222-226

37. Hau SC, Papastefanou V, Shah S, Sagoo MS, Restori M, Cohen V (2015) Evaluation of iris and iridociliary body lesions with anterior segment optical coherence tomography versus ultrasound B-scan. Br J Ophthalmol 99(1):81-86

38. Byrne SF (1986) Standardized echography of the eye and orbit. Neuroradiology 28(5-6):618-640

39. Huang LL, Hirose T (2012) Portable optical coherence tomography in management of vitreoretinal diseases: current developments, indications, and implications. Semin Ophthalmol 27(5-6):213-220

40. Elsheikh A, Geraghty B, Alhasso D, Knappett J, Campanelli M, Rama P (2010) Regional variation in the biomechanical properties of the human sclera. Exp Eye Res 90(5):624-633 